\title{
A Time Series Modeling and Prediction of Wireless Network Traffic
}

\author{
doi:10.3991/ijim.v3i1.284 \\ Gowrishankar and P.S.Satyanarayana \\ B.M.S. College of Engineering, Bangalore, India
}

\begin{abstract}
The number of users and their network utilization will enumerate the traffic of the network. The accurate and timely estimation of network traffic is increasingly becoming important in achieving guaranteed Quality of Service (QoS) in a wireless network. The better QoS can be maintained in the network by admission control, inter or intra network handovers by knowing the network traffic in advance. Here wireless network traffic is modeled as a nonlinear and non-stationary time series. In this framework, network traffic is predicted using neural network and statistical methods. The results of both the methods are compared on different time scales or time granularity. The Neural Network (NN) architectures used in this study are Recurrent Radial Basis Function Network (RRBFN) and Echo state network (ESN).The statistical model used here in this work is Fractional Auto Regressive Integrated Moving Average (FARIMA) model.
\end{abstract}

Index Terms-ESN, FARIMA, Nonlinear and nonstationary time series, Prediction method, Traffic flow.

\section{INTRODUCTION}

In recent past due to the technological breakthroughs in the field of wireless system has enabled the pervasive acceptance and deployment of wireless network. Still wireless system is not a preferred choice to the wired counterpart. The reason for preferring wired network to a wireless network is that wireless network will not ensure guaranteed QoS due to the unpredictable behavior of network traffic. The unpredictability is for the various factors such as user mobility, arrival pattern and diversified network requirement of user application. This leads to nonlinear and time varying traffic flow between wireless network infrastructure like wireless Access Points (AP)/Base Station (BS) and set of wireless devices using the network infrastructure. Now it is increasingly becoming important to provide guaranteed QoS to the users of the network, that can be achieved by one or more remedial measures like admission control, load balancing and intra or inter network handovers [1], for these measures accurate and timely forecasting of network traffic is the critical factor. The remedial measure in turn improves the QoS of a wireless network and hence the wireless network will be more reliable.

The nucleus of any prediction mechanism is to monitor the past and present behavior of the system and establish statistical relationship between set of inputs to the set of outputs over a given time scale or time granularity. This relationship is either linear or nonlinear and time invariant (stationary) or time variant (non-stationary). The wireless network traffic is a nonlinear and non-stationary system
[32]. The number of users and their network requirement is nonlinear due application characteristics and user behavior [2].The time varying behavior of the traffic is due to terminal mobility and network characteristics [3].The network applications are classified in to four classes namely conversational, streaming, interactive and background. The first two classes' network requirement is relatively constant and predictable. The third class of network requirement is based on the user behavior in the form of mouse clicks. The fourth and the last class network requirement is always on the state of the network [4]. Number of user and the amount of traffic generated by them has no correlation, hence the network traffic is nonlinear to the number users served by the wireless network [5].The number of users served by each AP or BS is dynamic due to the user preference, load balancing, inter/Intra network handover, admission control policy and network characteristics like fading, time-variant noise [3][6].Neural networks are the efficient methods to model, evaluate and predict the behavior of nonlinear and nonstationary systems[7].

In this work first data traffic time series is extracted from realistic wireless trace[8].The missing values of time series is estimated using Radial Basis function based Interpolation and function approximation method [9], then traffic time series is modeled and predicted using neural network architecture and finally results are compared with statistical model.

The rest of the paper is organized as follows. Section II, presents review of related work in the field of network traffic prediction and estimation. In section III, the system is modeled from traffic, prediction, statistical and neural network viewpoint. The system model is validated with set of forecasting trails in section IV. Finally, paper is concluded in section $\mathrm{V}$.

\section{RELEATED WORKS}

There are several research works on network traffic modeling and prediction of wireless network. Most of this will use network traces for the study; these trace data collected by three techniques, SNMP polling, Syslog or tcpdump from network sniffers [18]. The SNMP trace will specify total bytes transferred, number of bytes transferred with error and layer specific information of network [19]. The Syslog traces will enumerate the statistics of user behavior in terms of mobility and usage pattern of network. Sniffer will provide detail information about the size of packet, packet inter-arrival time and interface specific information of the network. The time granularity of SNMP, Syslog and tcpdump is in terms of few seconds, second and microseconds respectively. In [20], tcpdump 
and SNMP traces were used to study the variance of number of users, packet latency and handoffs between AP and laptops for the period of eight days. In [12], SNMP traces were used to characterize WLAN usage, user arrival and session duration, statistical model were proposed for network usage, arrival and session duration. In a study conducted at Dartmouth College [17], SNMP, Syslog and tcpdump traces were used to analyze the usage pattern and user distribution in a large wireless network. In [20], tcpdump trace is used to show the traffic characteristics from AP perspective and they also studied the behavior of traffic from Physical, Data link, IP and Transport Layer. Recently [21], SNMP trace is used to analyze the traffic characteristics like aggregate network load and its periodicity.

Some works have also been reported in the field of network traffic prediction. The prediction process can be classified as long and short term. In long term prediction the process of prediction time granularity is in the order of week and month. In short term prediction the time granularity is in terms of milliseconds, second, minutes and hours. The aggregated IP Backbone traffic is modeled for large time scale (weekly basis) and accurate forecasting for the period of six months by lower order ARIMA model [22]. In recent studies FARIMA process is used to model crucial features of self similar traffic like long range dependence (LRD) and short range dependence (SRD) of internet and broadband traffic, short term traffic forecasting in terms of millisecond to seconds of granularity were also reported in these works [23][24].In recent past[21], Normalized ARIMA Process and historical means of recent traffic were used to forecast hourly and daily trend of wireless traffic. The perceptron neural network model with back-propagation learning algorithm is the model used in the self similar traffic patterns [25]. Recently, Generalized Mean Neuron (GMN) model is used for forecasting of weekly internet traffic of a WAN router [26].

In the above works, the work is either limited to modeling of wireless traffic or forecasting of wire-line or wireless network traffic, both short term and long term trends. In wireless network the forecasting granularity is limited to hourly trend of the traffic and due to the large spikes and non-stationary behavior of time series of traffic, the series is smoothened and converted in to stationary series by applying power transformation technique. In the proposed work the wireless traffic is modeled as set of random processes and for the purpose of forecasting the wireless traces were used to construct time series and these time series were used to forecast traffic for single step and multistep step for second to minute time granularity.

\section{SYSTEM MODEL}

\section{A. Traffic Model}

In any stationary time series system, the series can be modeled as $y(t)=f\left(x_{t}\right)$, where $x_{t}=[y(t-1), y(t-2), \ldots y(t-m)]$. However this is not true in case of non-stationary time series, the entire series cannot be determined by single function $f($.$) , instead by set of function f_{1}, f_{2}, \ldots . . f_{k}$ where $k$ is the number of factors which determine each elements of the time series, hence the non-stationary time series can be modeled as

$$
\begin{gathered}
y(t)=\sum_{i=1}^{k} p_{i}^{t} f_{i}(t) \\
\sum_{i=1}^{k} p_{i}^{t}=1
\end{gathered}
$$

Where $f_{i}(t)$ is a random process and hence the nonstationary time series can be modeled by a set of random process [14].Since the traffic at AP or BS is a nonstationary time series, this can be determined by number of users and their arrival pattern, numbers of sessions(application) of individual user, session inter arrival and size of each session. All these are random processes [10].

$$
\operatorname{Tr}(t)=\sum_{i=1}^{3} p_{i}^{t} f_{i}(t)
$$

Where $f_{l}(t)$ is random process of number of users and the user arrival pattern, $\mathrm{f}_{1}(\mathrm{t})$ is discrete value continuous time process User distribution either uniform or lognormal process and user arrival pattern is time varying Poisson process[18][10][11]. The $f_{2}(t)$ is random process of number of session and session inter arrival pattern, $f_{2}(t)$ is discrete value and continuous time random process, user session is Lognormal process or time varying Poisson process and session inter arrival is a BiPareto, Weibull, Markov Modulated Poisson Process(MMPP) or Time varying Poisson process[10] [12][13]. The $f_{3}(t)$ is the size of each session, $f_{3}(t)$ is discrete value continuous time process and either Bipareto or Lognormal random Process[10][13].

\section{B. FARIMA Model}

There is a well established theory for statistical modeling and forecasting of stationary time series by Auto regressive Moving Average (ARMA) Process. As the order of Auto Regression(AR)value increases, the system can capture the seasonal trend of the time series and this leads to better approximation of stationary time series and the Moving Average(MA)value tries to remove effect of unknown initial value of the series[15]. The combination of AR and MA process will lead to high prediction accuracy of stationary time series with the removal of dependency of series by unknown initial values. However, in case of non-stationary time series such approximation will not be possible by simple ARMA process due to the fact that time series represents only one realization of set of stochastic process, for the better forecasting and approximation the time series can be decomposed into permanent (non-stationary) and transitory (stationary) parts [16].

$$
y(t)=y^{p}(t)+y^{k}(t)
$$

Where $y^{p}(t)$ denotes permanent and $y^{k}(t)$ is transitory component. Such decomposition will help in determining the Long Range Dependence (LRD) and Short Range Dependence (SRD) properties of the time series separately[15].A non-stationary process $\mathrm{y}(\mathrm{t})$ is 
transformed in to stationary process by differencing the process $d$ times, the parameter $d$ determines the LRD properties of the process

$$
(1-L)^{d} y(t)=\delta+x(t)
$$

Where $L$ is backward shift operator, $\delta=[y(t)-y(t-1)]$ and $x(t)$ is ARMA process, the parameter of ARMA process $\mathrm{p}$ and $\mathrm{q}$ determines SRD properties of the process. The original non-stationary process is denoted as $\operatorname{ARIMA}(p, d, q)$. The accuracy of non-stationary time series prediction lies on the estimation of differencing term $d$. If the degree of differencing $d$ is allowed to take non-integral value and the range is between $-1 / 2<d<1 / 2$, then such process is called as $F A R I M A(p, d, q)$ process[15]. When the non-stationary time series is modeled by $\operatorname{FARIMA}(p, d, q)$ process, the FARIMA $(p, d, q)$ model can be used to predict the future steps of time series[23].

$$
\begin{gathered}
\hat{y}(n)=\sum_{j=1}^{\infty} \pi_{j}^{n} y_{n-j} \\
\pi_{j}^{n}=\pi_{j+n-1}-\sum_{i=1}^{n-1} \pi_{i} \pi_{j}^{(n-1)} \\
\pi_{j}^{1}=\pi_{j} \\
\pi_{j}=\frac{(-1)^{j} \Gamma(1+d)}{\Gamma(1+j) \Gamma(1+d-j)}
\end{gathered}
$$

Where $\Gamma$ (.) is the gamma function and $\hat{y}($.$) is the$ predicted time series from estimated $\operatorname{FARIMA}(p, d, q)$ process.

\section{Neural Network Model}

Feed-forward neural network has the ability to map any nonlinear and non-stationary function to an arbitrary degree of accuracy [27].One such popular feed-forward network is the radial basis function network. It is a single hidden layer feed-forward network. Each node in the hidden layer has a parameter vector called as center. These centers are used to compare with network input and produce radically symmetrical response. These responses are scaled by connection weights of the output layer and then produce network output, where Gaussian basis function is used and given by

$$
\hat{y}=\sum_{i=1}^{n} w_{i} \exp \left(-\frac{\left\|y-\mu_{i}\right\|}{2 \sigma_{i}}\right)
$$

Where $\sigma_{i}$ is the dimension of the influence field of hidden layer neuron, $y$ and $\mu_{i}$ are input and prototype vector respectively. Radial Basis Function (RBF) has achieved considerable success in nonlinear function prediction but the performance of RBF is less satisfactory for the nonlinear and non-stationary function prediction [28]. The Recurrent Radial basis function network considers the time as an internal representation and the non-stationary aspect of nonlinear function can be obtained by having self-connection on the input neuron of sigmoidal firing function and the recurrent weights are in the range $[-1,+1]$, with normal distribution. This is a special case of locally recurrent, globally feed-forward neural network [29]. The RRBFN output for Gaussian basis function is

$$
\hat{y}(n)=\sum_{i=1}^{n} w_{i} \exp \left(-\frac{\sum_{j=1}^{m}\left(y^{j}-\mu_{i}^{j}\right)^{2}}{\sigma_{i}}\right)
$$

Where $\hat{y}($.$) is the predicted time series, \mathrm{n}$ is the number of step prediction and $\mathrm{j}$ is the number of neurons in the input layer of RRBFN system, the architecture of RRBFN model is shown in Figure 1.

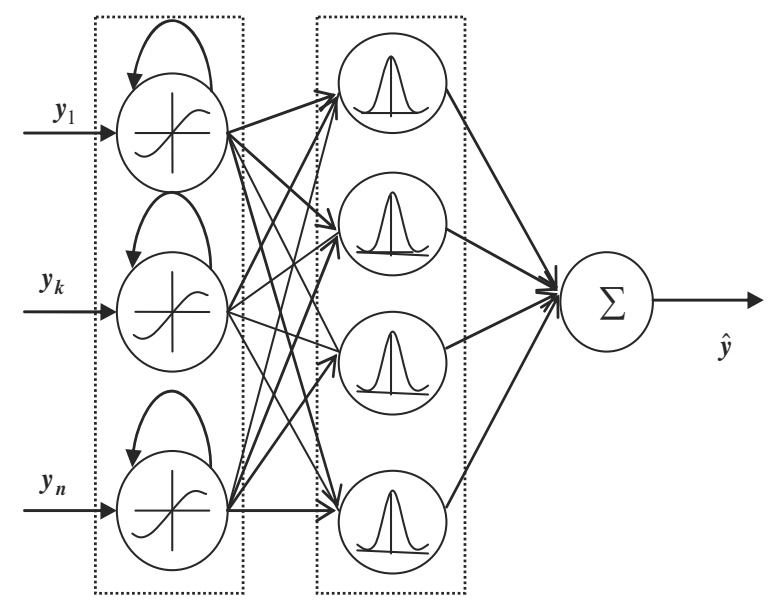

Figure 1. Recurrent Radial Basis Function Network

The Recurrent neural network with standard gradient decent algorithms will provide better function approximation for a short time step. For the longer temporal dependencies the gradient vanishes as the error signal is propagated back through time so that network weights are never adjusted correctly and the system will fail to predict for longer and complex time series steps. To deal with this an echo state network was proposed [30]. It consists of two parts such as a dynamical system with a rich set of dynamics followed by a memory-less output readout function shown in Figure 2. 


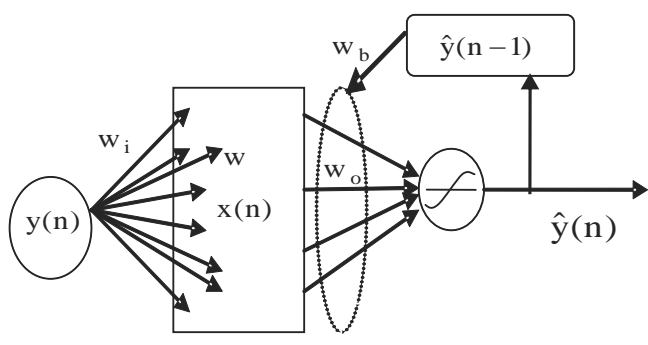

Figure 2. Echo State Networks

The dynamical system consists of large number of neurons that are randomly interconnected and selfconnected and these connections are fixed. This dynamical system is also called as reservoir and the optimal connections of neurons inside the reservoir will always require a number of trails. During the training process only weights of memory-less output function is changed through offline linear regression process or by online methods like recursive least square (RLS). The general state of reservoir is given by

$$
x(n)=\varphi\left(w_{i} y(n)+w x(n-1)+w_{b} \hat{y}(n-1)\right.
$$

Where $\varphi$ is the sigmoidal activation function, $y(n)$ is current input vector, $x(n-1)$ is the internal state of reservoir at time step $n-1, \hat{y}(n-1)$ is the output of ESN at previous time step. $w_{i}, w$ and $w_{b}$ are the input, reservoir and feedback weight vectors respectively. The output of ESN at time step $n$ is given by

$$
\hat{y}(n)=\varphi_{o}\left(w_{o} x(n)\right)
$$

Where $\varphi_{o}$ is the activation function of the output neuron and this can be linear or sigmoidal $w_{0}$ is the output weight vector and $x(n)$ is the current state of the reservoir.

\section{TRAFFIC PREDICTION}

The traffic prediction process involves two major phases, the first phase is time series extraction of data traffic and second phase is neural network and statistical model based time series prediction of wireless data traffic.

For the purpose of time series extraction of data traffic, the wireless trace available at CRAWDAD data repository is used [8]. The original dataset contains the traffic trace of 476 wireless APs for the period of 11 weeks [17], here the traffic prediction is limited to single AP for the time granularity of one second, ten second and one minute. The original trace file is filtered to obtain the traffic of single AP, for this SNMP trace is used to extract the detail traffic from tcpdump file. The filtered traffic trace is further processed to obtain amount of traffic in bytes per unit time. The filtered trace contains traffic between AP and set of wireless users for the period of one day. This traffic is combined and arranged to form a time series in to the different time scales such as 1 millisecond, 1 second and 1 minute. Once the time series is extracted from network trace, the traffic is forecasted for different time steps and the step refers to the unit of time. The filtered traffic has to be normalized for the purpose of n-step ahead prediction [31].

The Neural network based traffic prediction involves training and testing of RRBFN and ESN predictor. The time series extracted from trace used for training and testing of the predictors. The training and testing samples are randomly picked from the sample size of 1000 . The RRBFN network has three layers: input, hidden and output. Here 300 neurons in the input layer with sigmoidal activation function and with the recurrent connections, the range of recurrent weights are -1 to +1 . The hidden $\mathrm{RBF}$ layer has 175 neurons with RBF activation and output layer has single neuron with linear activation. The ESN network has 350 neurons in the reservoir with $75 \%$ of recurrent connection in the range of weights between -1 to +1 . Input weights are in the range of -0.40 to +0.40 and recurrent weights are in the range of -0.6 to +0.6 . Spectral radius is set to 0 and all the layer neurons have sigmoidal activation function, the predicted step sizes are 1 and 10 for both RRBFN and ESN.

In FARIMA based traffic prediction, The first step is to build FARIMA(p,d,q) model to describe wireless trace and the next step is to predict the network traffic for different time scale using FARIMA model . The process of building FARIMA model involves four stages [23]. First stage is to preprocess the normalized traffic to obtain zero mean time series. Next is to approximate the value d, where $\mathrm{d}=\mathrm{H}-1 / 2$, the value $\mathrm{H}$ refers to the Hurst Parameter, the Hurst Parameter is estimated by Variancetime plot of the normalized trace. Then to obtain the exact value of $d$ by fractionally differencing of $y(t)$ to obtain $x(t)$ of equation 3 , where $x(t)$ is ARMA model of the original time series. Finally ARMA process parameter $\mathrm{p}$ and $\mathrm{q}$, initially $\mathrm{p}$ and $\mathrm{q}$ values should start from smallest value, the best $(p, q)$ value is obtained by model identification and diagnostic checking process[15].The model parameters for various time granularity is listed in Table I. From the estimated FARIMA process and current and past values of time series, the future steps of time series are predicted. The predicted step sizes are 1 and 10.

TABLE I.

FARIMA MODEL PARAMETERS

\begin{tabular}{|c|c|c|c|c|c|}
\hline $\begin{array}{c}\text { Normalize } \\
\text { d Trace }\end{array}$ & $\mathbf{p}$ & $\mathbf{q}$ & $\mathbf{d}$ & Mean & Variance \\
\hline 1 Second & 4 & 2 & 0.0178 & 0.0126 & 0.00067 \\
\hline 10 Seconds & 4 & 2 & 0.1865 & 0.1246 & 0.0036 \\
\hline 1 Minute & 4 & 2 & 0.3457 & 0.4101 & 0.0065 \\
\hline
\end{tabular}


The Figure 3 and 4 shows the 1 step and 10 step traffic predictions from RRBFN, ESN and FARIMA model, for 1 second time granularity. The 1 step and 10 step traffic

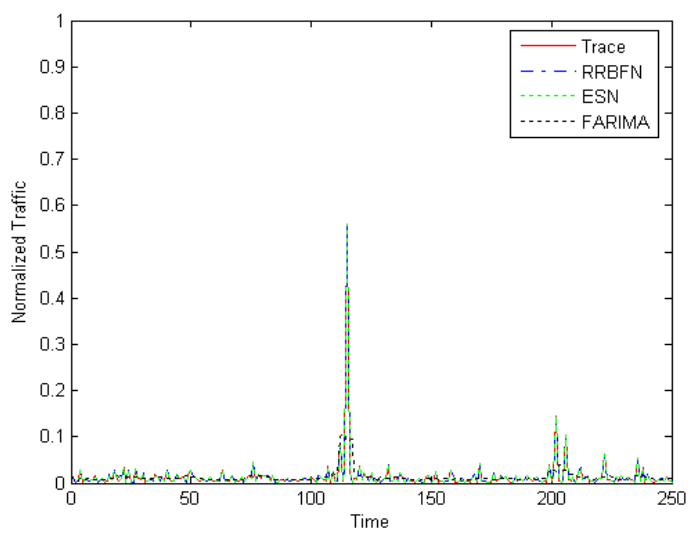

Figure 3. 1-Step Traffic predicition (1-Second Interval)

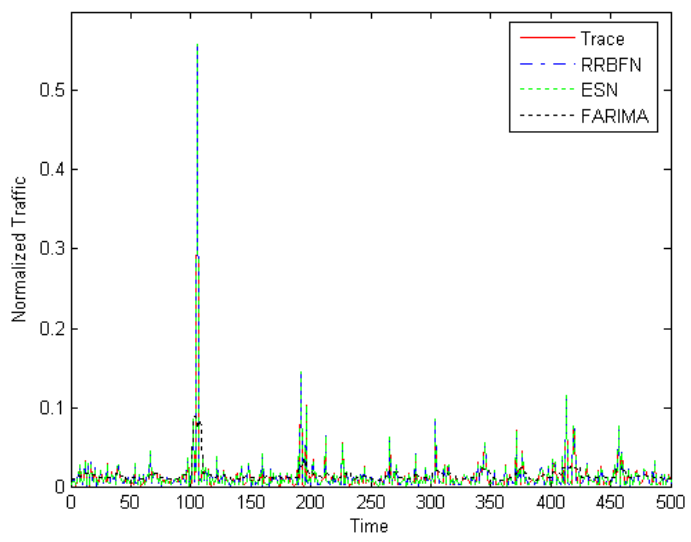

Figure 4. 10-Step Traffic predicition (1-Second Interval)

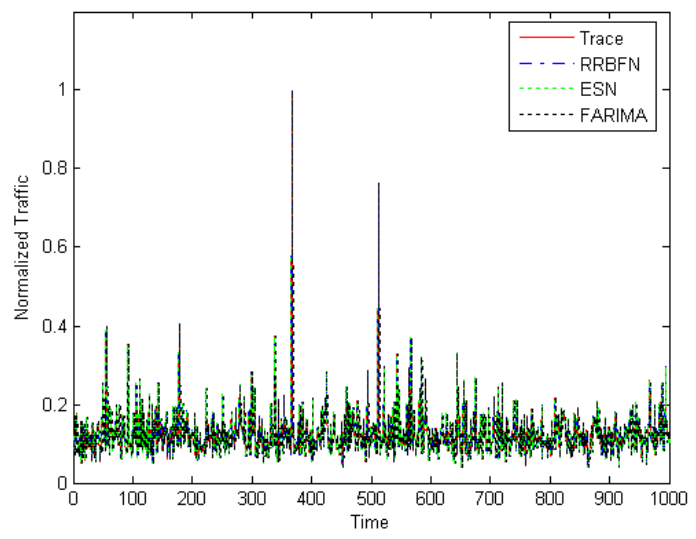

Figure 5. 1-Step Traffic predicition (10-Second Interval) predictions for 10 second time granularity are shown in Figure 5 and 6. The Figure 7 and 8 shows 1 and 10 step prediction for the time granularity of 1 minute.

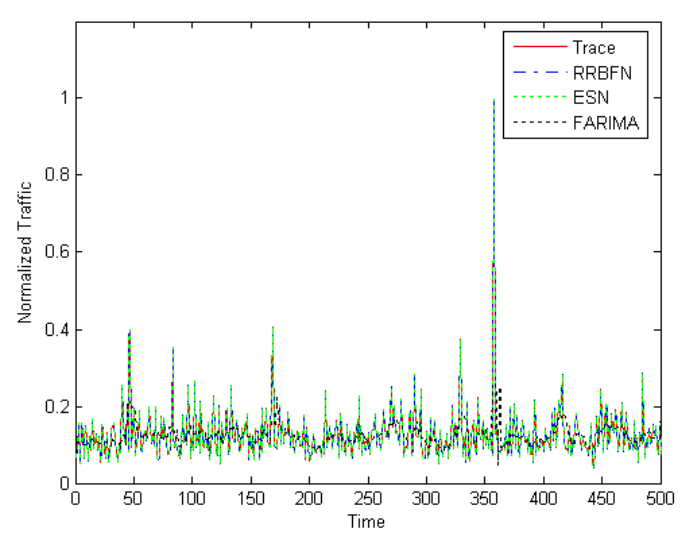

Figure 6. 10-Step Traffic predicition (10-Second Interval)

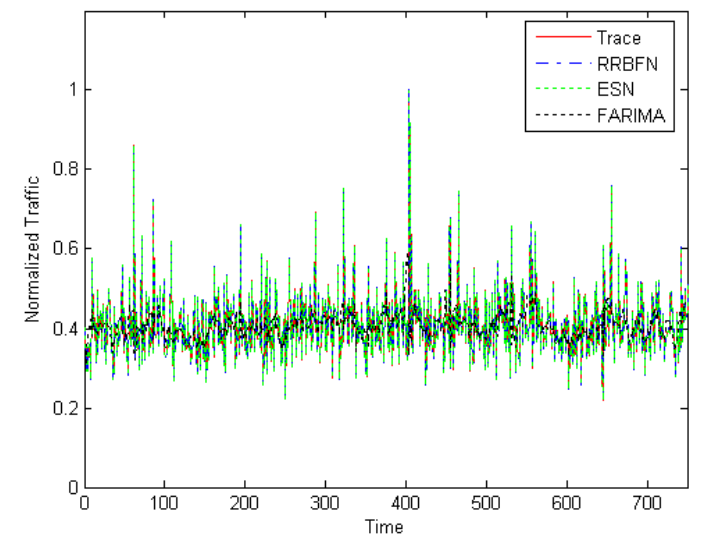

Figure 7. 1-Step Traffic predicition (1-Minute Interval)

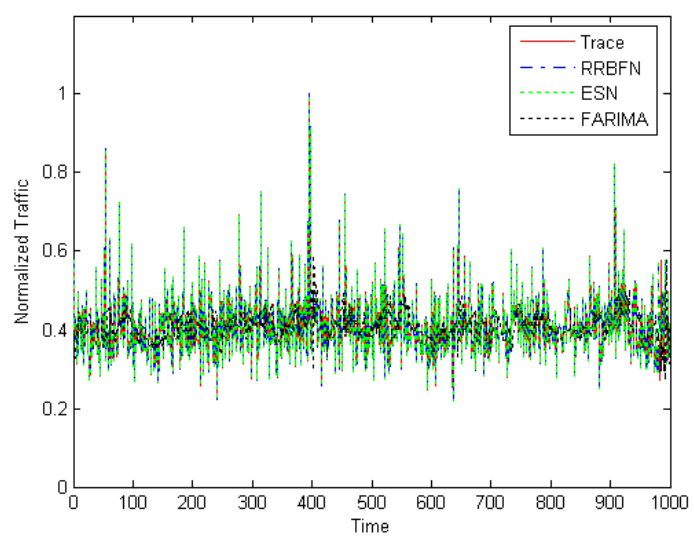

Figure 8. 10-Step Traffic predicition (1-Minute Interval) 
Complementary-CDF (CCDF) plot for trace and predicted samples for 1 step and 10 step granularity for 1 second granularity shown in Figure 9 and 10. CCDF plot

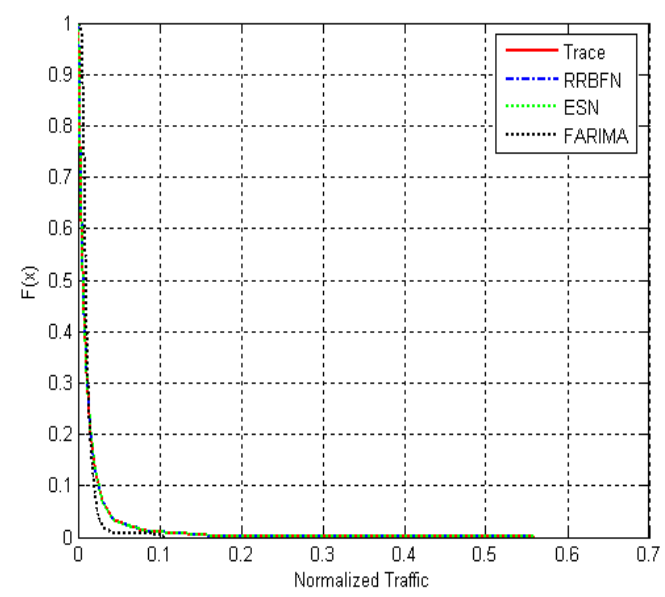

Figure 9. 1-Step CCDF Plot (1-Second Interval)

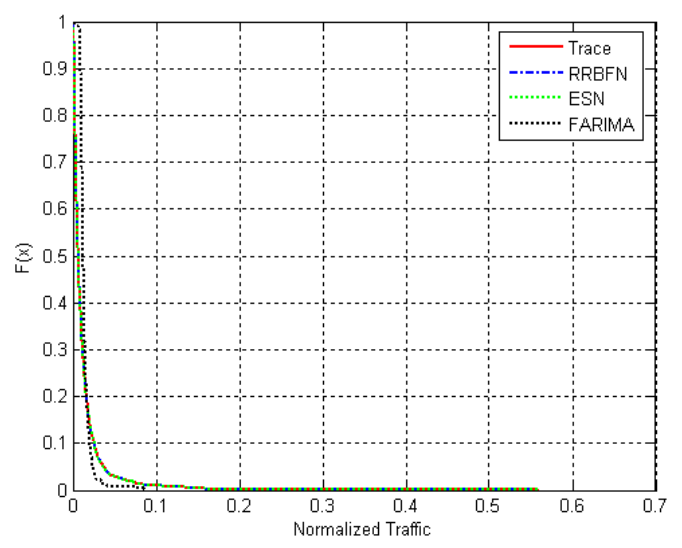

Figure 10. 10-Step CCDF Plot (1-Second Interval)

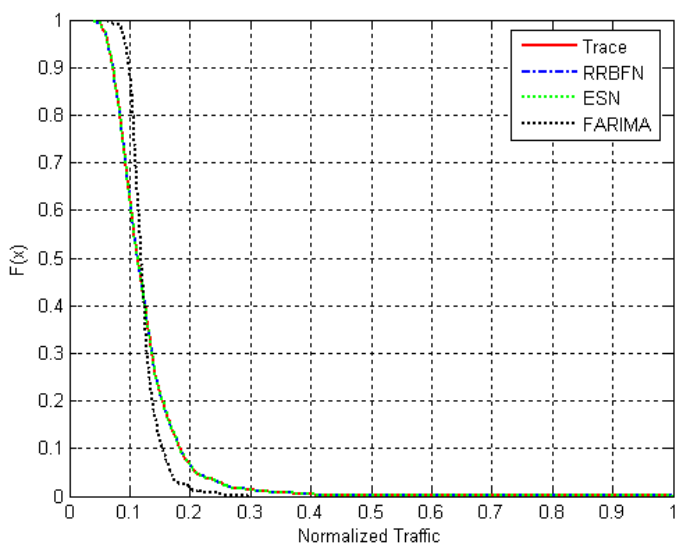

Figure 11. 1-Step CCDF Plot (10-Second Interval) for model output and trace for 10 second granularity is shown in Figure 11 and 12. Figure 13 and 14 shows 1 and 10 step predicted output for 1 minute granularity.

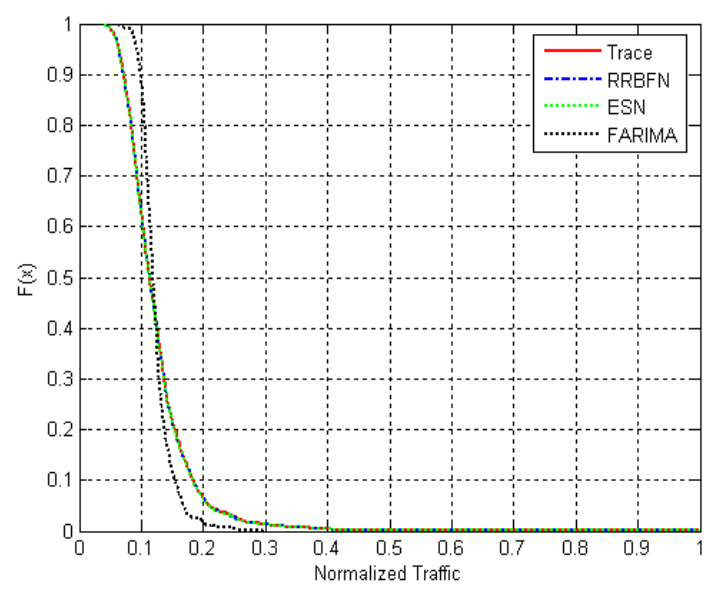

Figure 12. 10-Step CCDF Plot (10-Second Interval)

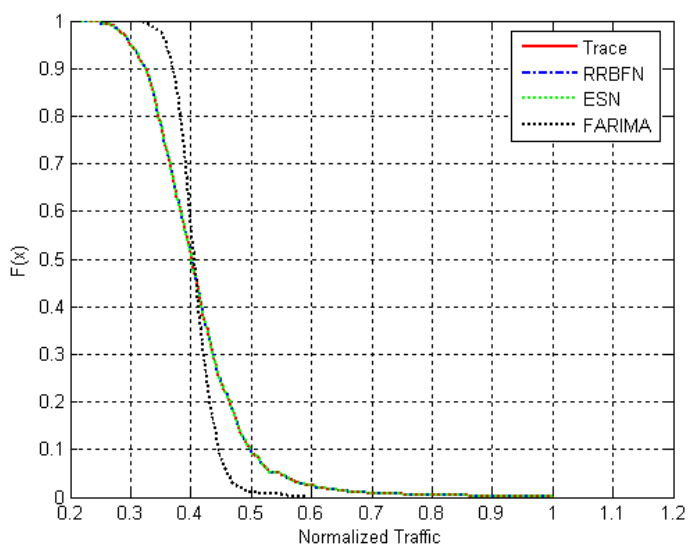

Figure 13. 1-Step CCDF Plot (1-Minute Interval)

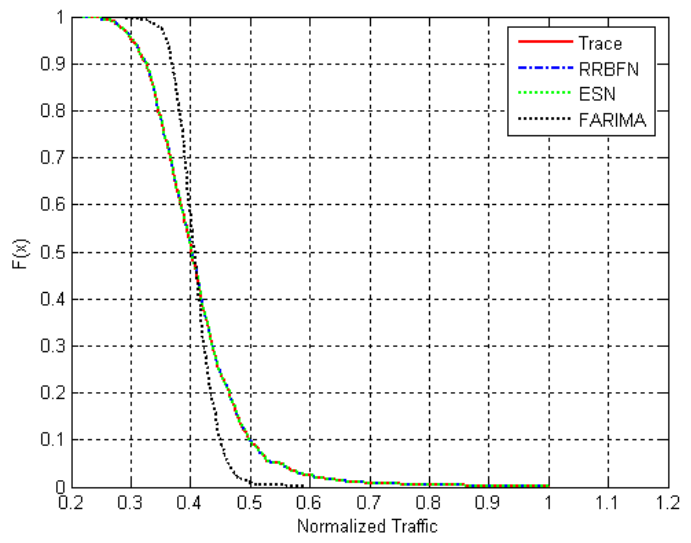

Figure 14. 10-Step CCDF Plot(1-Minute Interval) 
The Quantile-Quantile Plot (Q-Q Plot) RRBFN, ESN, FARIMA Models output and trace for 1 and 10 steps for 1 second granularity is shown in Figure 15 and 16 Figure

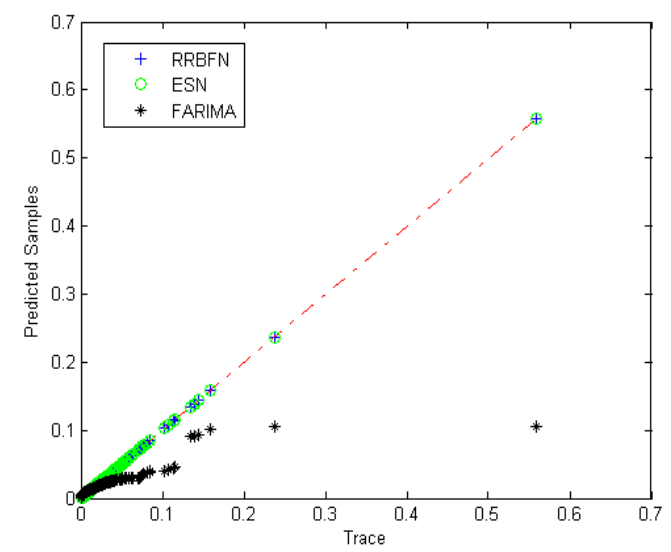

Figure 15. 1-Step Q-Q Plot (1-Second Interval)

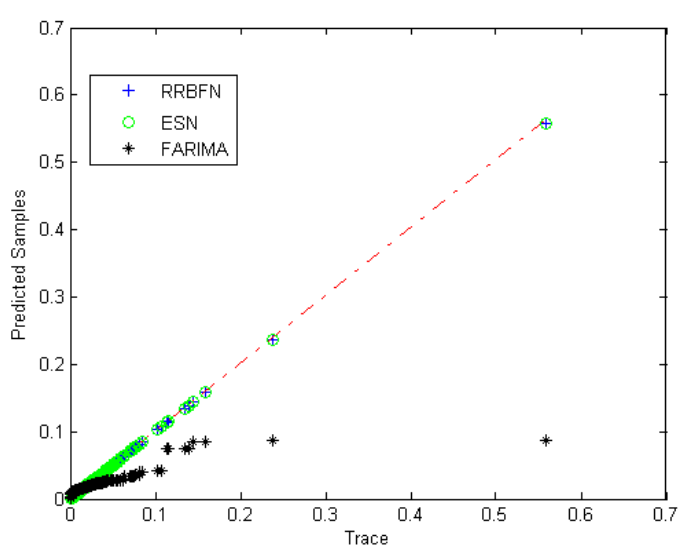

Figure 16. 10-Step Q-Q Plot (1-Second Interval)

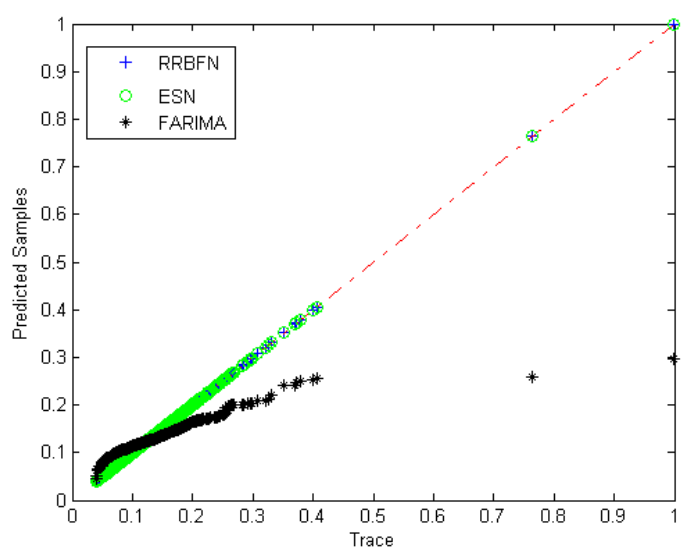

Figure 17. 1-Step Q-Q Plot (10-Second Interval)
17 and 18 is the Q-Q plot for 1 and 10 step for 10 second granularity. The Q-Q plot for 1 and 10 step prediction for 1 minute granularity is shown in Figure 19 and 20.

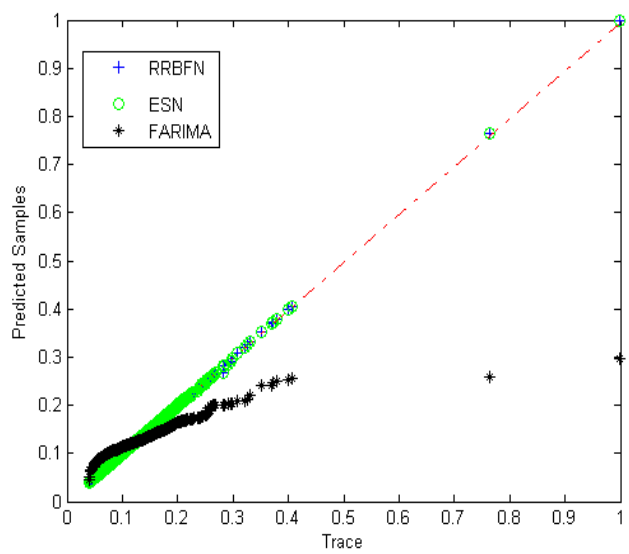

Figure 18. 10-Step Q-Q Plot (10-Second Interval)

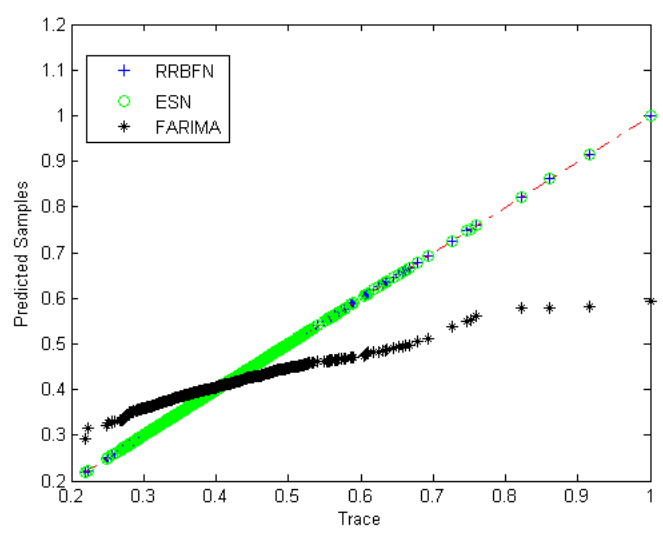

Figure 19. 1-Step Q-Q Plot (1-Minute Interval)

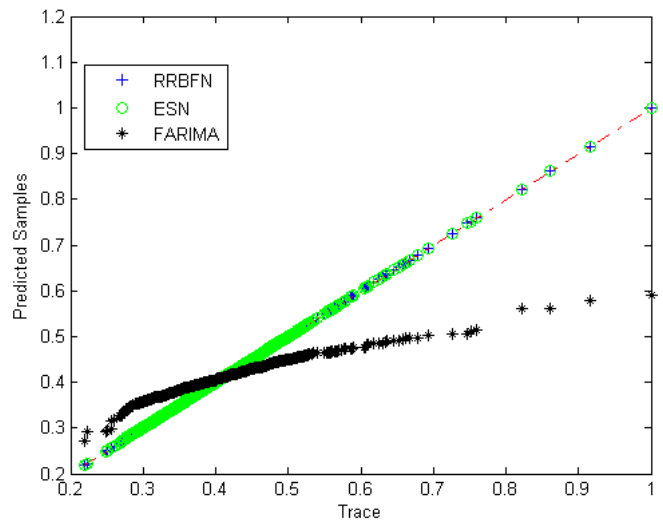

Figure 20. 10-Step Q-Q Plot(1-Minute Interval) 
Normalized Auto Correlation Function (ACF) plot for trace and predicted samples for 1 step and 10 step granularity for 1 second granularity shown in Figure 21 and 22. ACF plot for model output and trace for 10 second granularity is shown in Figure 23 and 24. Figure 25 and 26 shows ACF plot for 1 and 10 step prediction output for 1 minute granularity.

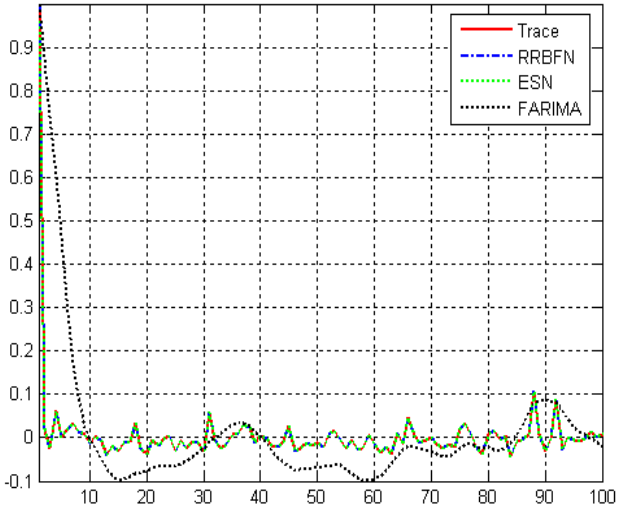

Figure 21. 1-Step ACF Plot (1-Second Interval)

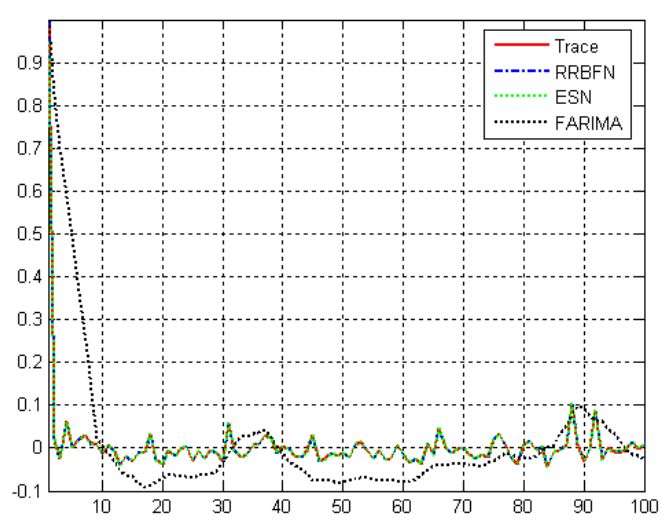

Figure 22. 10-Step ACF Plot (1-Second Interval)

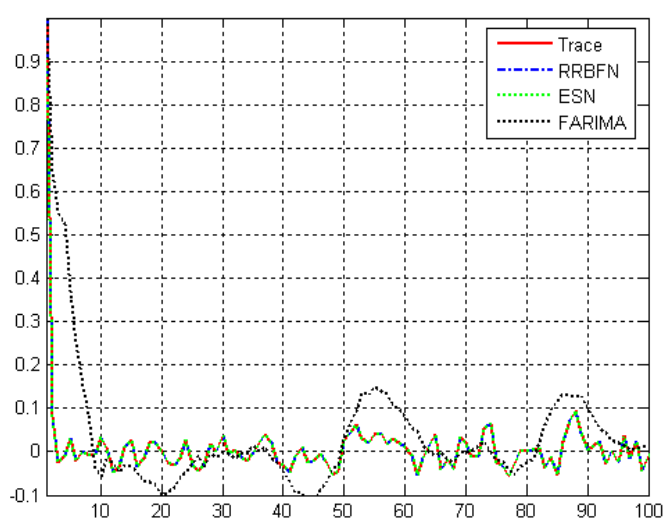

Figure 23. 1-Step ACF Plot (10-Second Interval)

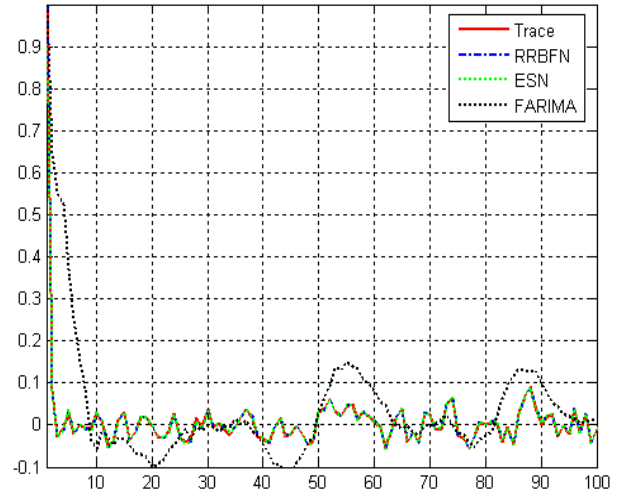

10-Step ACF Plot (10-Second Interval)

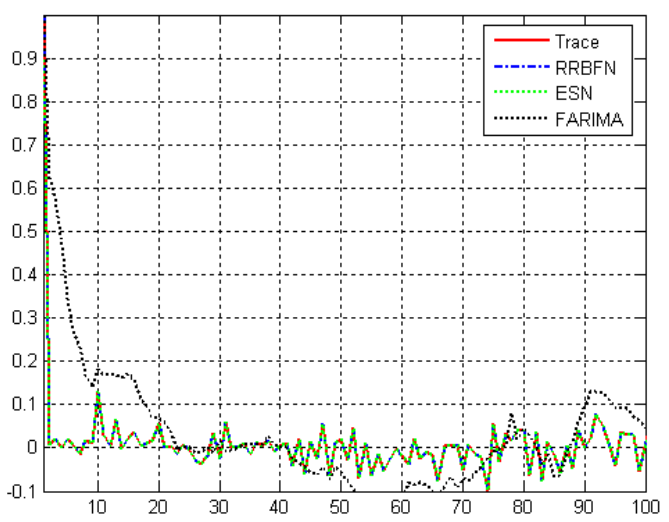

Figure 24. 1-Step ACF Plot (1-Minute Interval)

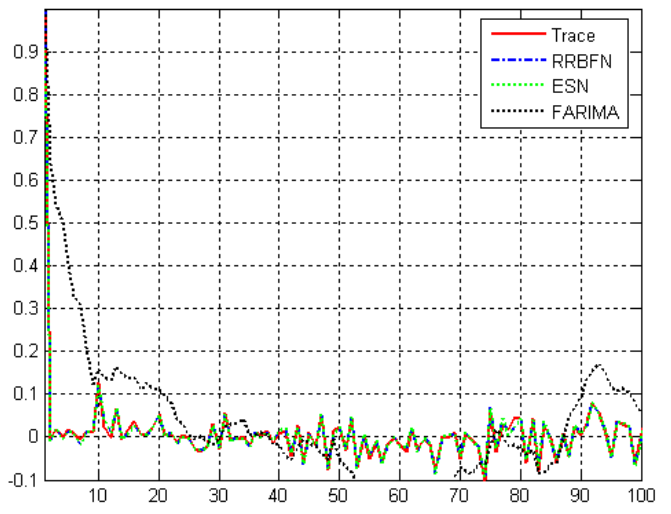

Figure 25. 10-Step ACF Plot(1-Minute Interval) 
From CCDF plot, the functional behavior of trace and model output can be visually determined. The $\mathrm{Q}-\mathrm{Q}$ plot and ACF plot the first and second order statistical relationship between normalized trace and predicted output of neural and statistical models can be visually determined [10].

\section{CONCLUSION}

In this paper wireless network traffic for short time step is modeled as time series and the future values of time series are predicted by RRBFN, ESN and FARIMA models. The prediction accuracy of both Neural network model found to be superior for 1 second, 10 seconds and 1 Minute time granularity, The Prediction accuracy of RRBFN model for three time granularity ranges from $96.4 \%$ to $98.3 \%$. Prediction accuracy of ESN model for three time granularity found to be $97.8 \%$, to $98.1 \%$. The prediction accuracy of FARIMA model of three time granularities seems to be less satisfactory compared to neural models and the prediction accuracy is the range of $78.5 \%$ to $80.2 \%$. The FARIMA prediction accuracy will be slightly increased in 1 minute granularity. This is due to the fact that the time aggregation makes the wireless trace exhibits high degree of self similarity. The estimated Hurst Parameter $H$ from variance time plot is 0.8457.In a shorter time granularity time series behavior is nonlinear and non-stationary due to burstiness in the network traffic and user behavior.

In neural Network Models, ESN based predictor is slightly better in terms of prediction accuracy, but network takes more training samples and longer training time compared to RRBFN. During the training phase the training Mean Squared Error (MSE) for ESN and RRBFN are 0.01254 and 0.0162 respectively.

In general, the results show that neural network predictors are better than statistical models. The performance of FARIMA model is based on parameter estimation, where the process of parameter estimation is based on trial and error which affects the performance of FARIMA model to the large extent. The property of burstiness of wireless network traffic will be better captured by neural network predictors, the long range dependency is captured by adjusting the weights of the neurons during the training phase, Short range dependency the input is confined by the recurrent architecture of the network.

Future work includes applying NN models for long term prediction of wireless traffic. Furthermore, the NN based predictors are used for Available Bandwidth Prediction (ABW) and Bit Error Rate (BER) prediction for the purpose of inter and intra network handovers, admission control in heterogeneous wireless network infrastructure.

\section{ACKNOWLEDGMENT}

The authors wish to thank the editor and anonymous reviewers for their valuable comments and suggestions that helped to improve the presentation of this paper. The authors also wish to thank Dr.G.N.Sekhar, HOD Mathematics for the mathematical framework of this research work. The authors wish to acknowledge the constant support during the research work by Principal, Chairman and Board of Management B.M.S. College of Engineering.

\section{REFERENCES}

[1] W.Xiucho and A.L.Ananda, "Link Characteristics Estimation for 802.11 DCF Based WLAN", $29^{\text {th }}$ Annual IEEE International conference on Local Computer Network, pp. 302-309, November 2004.

[2] H. Choi and J.Limb, “A Behavioral Model of web Traffic”, $7^{\text {th }}$ International conference on network Protocols, pp. 327-334, November 1999.

[3] W.Hsu and A.Helmy, "On modeling user Association in Wireless LAN Traces on University Campus”, $2^{\text {nd }}$ IEEE International workshop on wireless network measurement, pp 1-9, April 2006.

[4] ETSI, "Universal Mobile Telecommunication Systems(UMTS);Selection Procedures for the Choice of radio Transmission Technologies of the UMTS”, Technical Report TR 101112 v. 3.2.0, 1998

[5] J. Lee, S. Choi and H.W. Jung, "Analysis of User Behavior and Traffic Pattern in a Large-Scale 802.11 a/b Network", $1^{s t}$ IEEE International workshop on wireless network measurement, pp 1-8, April 2005.

[6] J.Landman and P.Kritzinger, "Delay Analysis of downlink IP Traffic on UMTS Mobile” ,Performance Evaluation, Vol. 62, (14),pp. 68 -82, October 2005. (doi:10.1016/j.peva.2005.07.007)

[7] Y.K.Tay, J.O.Kyong, K.Chiho and et.al, "Artificial Neural Network for non-stationary time series”, Neurocomputing, Vol. 61, pp. $439-447$, October 2004. (doi:10.1016/j.neucom.20 04.04.002)

[8] “CRAWDAD”, http://crawdad.cs.dartmouth.edu , November 25, 2007.

[9] S.A.Sara, "Integrated Multiquadric Radial Basis Function Approximation Methods", Computers and Mathematics with Applications, Vol. 51, No.8, pp.1283-1296, 2006 (doi:10.1016/j.camwa.2006.04.014)

[10] H.Felx, K.Merkouris P.Maria and et.al, "Spatio-Temporal Modeling of Traffic Workload in a campus WLAN", $1^{\text {st }}$ International workshop on Technology and policy for Accessing Spectrum, August 2006

[11] M.George, H.Y.W.Strasky, Y.Yuan and L.Songwu, "Characterizing flows in Large Wireless Data Network", $10^{\text {th }}$ International Conference on Mobile Computing and Networking, September 2004.

[12] B.Anand, M.V.Geoffrey, P. Bahal and P.Venkatragan, "Characterizing the user Behavior and network performance in public wireless LAN”, ACM Sigmetrics , pp 195-205,June 2002.

[13] J.N.Carl, S.Iraj, S.Wim, “A compounded model for TCP connection arrival for LAN and WAN Applications”, Computer Networks, Vol. 40, No. 3, pp 319-337, October 2002. (doi:10.1016/S1389-1286(02)00298-0)

[14] R.Dahlhaus, "Fitting Time Series Models to Non-stationary Processes”, The Annals of Statistics, Vol.25 No.1, pp 1-37, February 1997. (doi:10.1214/aos/1034276620)

[15] K.Gebhard and W.Jurgen, "Introduction to Modern Time Series Analysis”, Springer-verlag, 2007.

[16] R.N.Charls and K.Heejoon, "Spurious periodicity in inappropriately Detrended Time Series”, Econometrica, Vol.49, pp.741 -751, 1981. (doi:10.2307/1911520)

[17] K.David and E.Kobby, "Analysis of Campus wide wireless network”, Wireless Network, Vol. 11, pp. 115 -113, January 2005. (doi:10.1007/s11276-004-4750-0)

[18] S.Rajeev, A.L.Ananda, C.C.Mun and et.al. "Mobile, Wireless and Sensor Networks: Technology, Applications and Future Directions”, John Wiley and Sons, Inc, 2006.

[19] IEEE computer society, "LAN MAN standards committee IEEE 802.11 Management Information Base in IEEE std. 802.11-1999”, 1999

[20] Y.Jihwang, Y.Moustafa. and A.Ashok, "Characterizing the IEEE 802.11 Traffic: The Wireless Side”, International workshop on wireless traffic measurement and modeling, 2005

[21] P.Maria, S.Haipeng, R.Elias and et.al, "Short-term traffic forecasting in a campus-wide wireless network", $16^{\text {th }}$ IEEE International symposium on Personal, indoor and Mobile Radio communication, pp.1446-1452, September 2004. 
[22] P.Konstantina, T.Nina, Z.Zhang and et.al, "Long-term Forecasting of Internet Backbone Traffic", IEEE Transaction on Neural Networks, Vol. 16, No.5, pp. 1110-1124, June 2005. (doi:10.1109/TNN.2005.853437)

[23] S.Yantai, J.Zhigang, Z.Lainfang and et.al, "Traffic Prediction Using FARIMA Models”, IEEE International Conference on Communication, Vol.2, pp. 891-895, June 1999.

[24] M.R.Corradi, G.Garroppo S.Giordano and et.al, "Analysis of fARIMA processes in the modeling of broadband traffic", IEEE International Conference on Communication, Vol.3 pp.964-968, June 2001.

[25] H.Yousefizadeh, “ Neural Network Modeling of ON-OFF Source Models with Self Similar Characteristics”, $1^{\text {st }}$ Workshop on Fractals and Self-Similarity, ACMSIGKDD, July 2002

[26] R.N.Yadav., K.K.Prem and J.Joseph, "Neural network learning with generalized-mean based neuron model”, soft computing- $A$ fusion of foundations, Methodologies and Application, Vol.10, No. 3, pp. 257-263, February 2006.

[27] M.Li, G.Huang, P.Saratchandran and N.Sundarajan, "Performance Evaluation of GAP-RBF Network in Channel Equalization", Neural processing Letters, Vol. 22, No. 2, pp.223-233, October 2005. (doi:10.1007/s11063-005-6799-x)

[28] S.Haykin, "Neural Networks: A Comprehensive Foundation”, Person Education Inc., 1999.
[29] R.Zemouri, D.Racoceanu and N.Zerhouni, "Recurrent Radial Basis Function Network for Time Series Prediction”, Engineering Applications of Artificial Intelligence, Vol. 16, No.5-6. pp. 453463, August - September 2003. (doi:10.1016/S0952-1976(03)000 63-0)

[30] H.Jaeger and H.Haas, "Harnessing nonlinearity: Predicting chaotic systems and saving energy in wireless communication”, Sciences 2, Vol. 304, No.5667, pp 78-80, April 2004.

\section{AUTHORS}

Gowrishankar is with the Computer Science and Engineering Department, B.M.S. College of Engineering, Visvesvarya Technological University, P.O. Box. 1908, Bull Temple Road, Bangalore-560 019, Karnataka, INDIA (e-mail: gowrishankar.cse@bmsce.ac.in)

P.S.Satyanarayana is with the Computer Science and Engineering Department, B.M.S. College of Engineering, Visvesvarya Technological University, P.O. Box. 1908, Bull Temple Road, Bangalore-560 019, Karnataka, INDIA (e-mail: pss.ece@bmsce.ac.in).

This work was supported in part by the Technical Education Quality Improvement Program (TEQIP) funds, Government of India. Manuscript received 09 April 2008. Published as submitted by the authors. 